\title{
Effect of enhanced ionizing radiation on the cloud electricity after the Fukushima nuclear accident
}

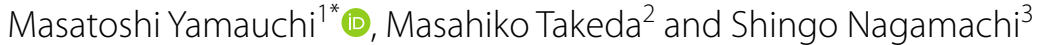

\begin{abstract}
The vertical downward component of the DC atmospheric electric field, or potential gradient (PG), at Kakioka $150 \mathrm{~km}$ southwest of the Fukushima Dai-ichi Nuclear Power Plant (FNPP1) was analyzed before and after the FNPP1 accident to examine possible influence of floating radioactive particles on the PG under the highly electrified clouds. Using 1-min PG data from March 13 to April 30 (late April 2011 corresponding to the time when floating radioactive materials decreased significantly) from 2006 to 2015, time profiles to/from the PG peaks (<- 100 or >+ 100 V/m) for 2011 are compared with other years. The PG developed toward and decays from its negative peaks faster in the first 50 days after the FNPP1 accident than for the same period in other years, making the 10-min averaged PG values for the same negative PG peak higher (smaller in terms of absolute value) during the first 50 days after the FNPP1 accident than those in other years in the study period, while the distribution of peak PG values is similar between 2011 and the other years. The observed shortening of the timescale is symmetric between the rise and decay and is the most clear when the negative PG peak is about - 200 to $-400 \mathrm{~V} / \mathrm{m}$. For positive peaks, the change of the baseline resulting from the radioactive contamination on the ground in 2011 caused superficial difference on such time profiles. Otherwise, there are no significant difference between 2011 and the other years. Possible explanations based on increased ionizing radiation from floating radioactive particles, which are found to be concentrated at low altitudes $(<500 \mathrm{~m}$ according to radiosonde in-situ observations of gamma rays) where negative cloud charges normally exceed positive charges, are discussed. The scenarios discussed include enhanced electrostatic shielding and electric conductivity-led decay rate of cloud charges. The result opens up a new possibility of using PG as an independent monitor of radioactivity at some altitudes in case of nuclear accidents.
\end{abstract}

Keywords: Radioactive aerosol, Fukushima nuclear accident, Atmospheric electricity, Cloud charges, Electric shielding

\section{Introduction}

The accident at Fukushima Dai-ichi Nuclear Power Plant (FNPP1) in March 2011 released massive quantities of radionuclides, including more than $10^{16} \mathrm{~Bq}$ of ${ }^{137} \mathrm{Cs}$ alone, to the atmosphere (NISA 2011). They emit ionizing radiation during their decay process and thus increase atmospheric ion density $\left(n_{i}\right)$ and electric conductivity $(\sigma)$, particularly near the ground where radioactive fallout accumulates. Such a local increase in $\sigma$ suppresses the

\footnotetext{
*Correspondence: M.Yamauchi@irf.se

1 Swedish Institute of Space Physics, Box 812, 98128 Kiruna, Sweden

Full list of author information is available at the end of the article
}

vertical (downward) component of the DC electric field, or potential gradient (PG), near the ground (e.g., Harris 1955; Tuomi 1988; Markson 2007; Rycroft et al. 2008; Williams 2009). This phenomenon were also observed at Kakioka, $150 \mathrm{~km}$ southwest of the FNPP1, after the radioactive fallout on March 14, 2011; the ground-level PG under fine weather conditions at Kakioka decreased by more than one order of magnitude (Takeda et al. 2011; Yamauchi et al. 2012).

Before the FNPP1 accident, only the effect of the increased $\sigma$ near ground on positive PG under fine weather was studied. However, no estimation of the 
thickness of increased $\sigma$ nor influence on negative PG under electrified clouds was discussed. If one can use PG to estimate the increased $\sigma$ values by floating radioactive particles in the air, that would become a useful remote sensing clue for understanding the atmospheric transport of radioactive particles, particularly after accidents at nuclear plants.

Yamauchi et al. (2012) used the temporal variation of the PG under fine weather to estimate the motion (fallout, floating near ground, and re-suspension) of radioactive particles by comparing this data with the radiation dose rate, weather, and wind at ground level. These comparisons revealed that (a) the dry deposition on March 15 caused significant re-suspension, (b) the wet deposition starting from March 20 caused temporary settlement, but ready for re-suspension until late April after the land dried out, and (c) the accumulated radioactive particles on canopies dropped to the ground during heavy rain on and April 8 and 18. These indications mean that substantial quantities of radioactive particles were floating during first 50 days after the FNPP1 accident, as confirmed by the direct measurements both at a fixed station (Kanai 2012), samples (Kita et al. 2013), and balloon in situ measurements (Fukushima University 2011). Thus, PG data can also be used to estimate the motion of floating radionuclides in the air.

So far we have analyzed positive near-quiet PG data to estimate the distribution and dynamics of radionuclide. The next obvious question is whether floating radionuclides (or increased conductivity $\sigma$ ) causes any detectable effects on negative PGs, i.e., on the point-charge electric field under the electrified clouds. Israelsson et al. (1987) suggested that the electrical characteristics of raindrops or cloud nuclei might be affected. However, the effect of increased $\sigma$ on the cloud-origin negative PG has never been considered.

One obvious candidate is (1) shortening of the charge-loss (discharge) time $\left(\tau_{\mathrm{d}}\right)$ of cloud charges by the increased conductivity or ion density $\left(\tau_{\mathrm{d}} \propto \sigma^{-1} \propto n_{i}^{-1}\right)$. Here, we consider ion production by enhanced gamma rays from radionuclides (Fig. 1b) inside and near the cloud, and the discharge is mainly within the cloud without lightning. The gamma ray enhancement in the atmosphere was actually observed by the radiosonde measurements at Fukushima (Fukushima University 2011). They showed a notable enhancement of gamma rays at altitudes of $<500 \mathrm{~m}$ and $7-8 \mathrm{~km}$.

In addition to the increased $\sigma$-led discharge rate, we also expect electrostatic suppression of point-charge electric fields by the increased ion mobility in the electric field, as illustrated in Fig. 1, similar to the processes of either (2) Debye shielding or (3) dielectric media.

In the Debye shielding theory (Debye and Huckel 1923) that uses statistical thermodynamics, an isolated charge

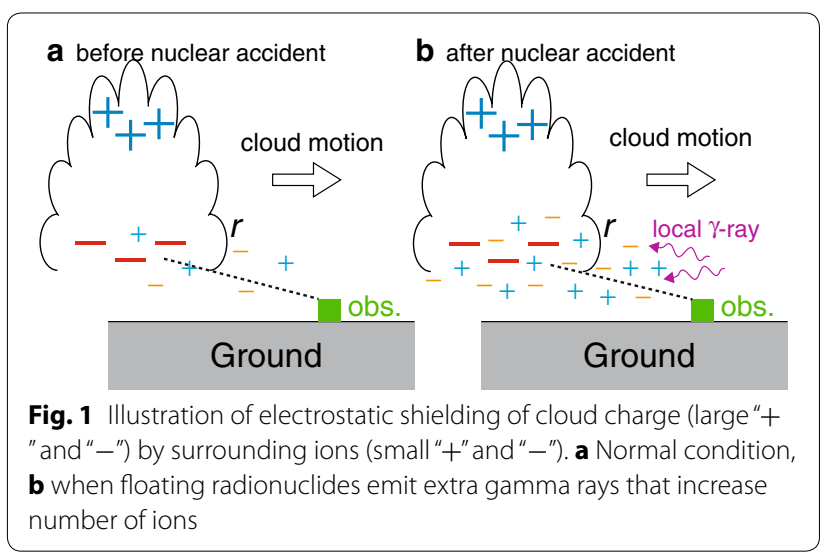

will be surrounded by oppositely charged ions unless a strong external force regulates the charge distribution. However, under electrified clouds, the atmospheric electric field reaches $10^{3}-10^{4} \mathrm{~V} / \mathrm{m}$, providing a much higher potential energy than thermal energy at only $1 \mathrm{~mm}$ from single charges. [This is the mean distance between the atmospheric ions under average atmospheric ion density of $10^{9} \mathrm{~m}^{-3}$ (e.g., Harrison and Carslaw 2003).] In such a case, the thermal re-distribution of ions (Debye shielding) is not as effective as re-distribution by the external electric field.

Since the re-distribution of ions is strongly bounded by ion-neutral collisions in the atmosphere, we expect some electrostatic polarization with only small increase in the electric current. As the result, the electric field decreases for the same point charges, like a dielectric media (or condenser). Such a condenser effect has two timescales: One is regulated by the conduction current (large-scale motion), with expected timescale of few tens minutes in the lower atmosphere under normal conditions. The other is regulated by individual ion reactions to the changing electric field and is in the order of plasma frequency with mass of ions $\left(\propto n_{i} / m_{i} \sim 10^{3} \mathrm{~Hz}\right)$, which is much smaller than the collision frequency. The shielding effect might already appear in the timescale of the PG measurement because of the second effect. In such a case, the effect is expected to be nearly proportional to the total numbers of ions distributed between the electrified cloud and the ground, and hence to the density $n_{i}$.

All three mechanisms predict the suppression of ground-level PG for the same cloud charges. The next question is whether the predicted suppression of PG is detectable. Figure 2 shows examples of the PG behavior during rainfall events after the FNPP1 accident (March 15, 2011) and during a similar event with the rain strength, PG amplitude, and time of year before the accident (March 23, 2010). The temporal resolution of the rain measurement (accumulation method) is 10 
min. The PG variation in Fig. 2a abruptly stopped when the rain ended $(\sim 20: 15 \mathrm{UT})$, but continued after 13:50 UT when the rain stopped in Fig. 2b, suggesting that the increased atmospheric radioactivity might have caused some detectable effects on PG. Such an effect has never been studied before, either after nuclear tests (Harris 1955) or the Chernobyl nuclear accident (Israelsson and Knudsen 1986; Tuomi 1988). Therefore, it is worth finding any statistical differences in the behavior of PG, particularly the negative peak values and timescale around it, between during the first 50 days after the Fukushima accident (when the atmospheric radioactivity increased) and the same time in the other years. This is the purpose of this paper.

\section{Data and analysis}

We use 1-min resolution PG data from Kakioka from 2006, since the digital data are publicly available from that year. The sensor uses Masacart's insulated waterdropper collector, placed $2.55 \mathrm{~m}$ above the ground and $1.17 \mathrm{~m}$ away from the wall inside a house, with water dropping outside this building (Shigeno et al. 2001). The insulation was tested and confirmed even during rainfall events as part of maintenance. The sampling rate is $1 \mathrm{~Hz}$ with a calibrated system response time of a few seconds for grounding and about a minute for $-700 \mathrm{~V} / \mathrm{m}$ level PG. Furthermore, the system detected more than
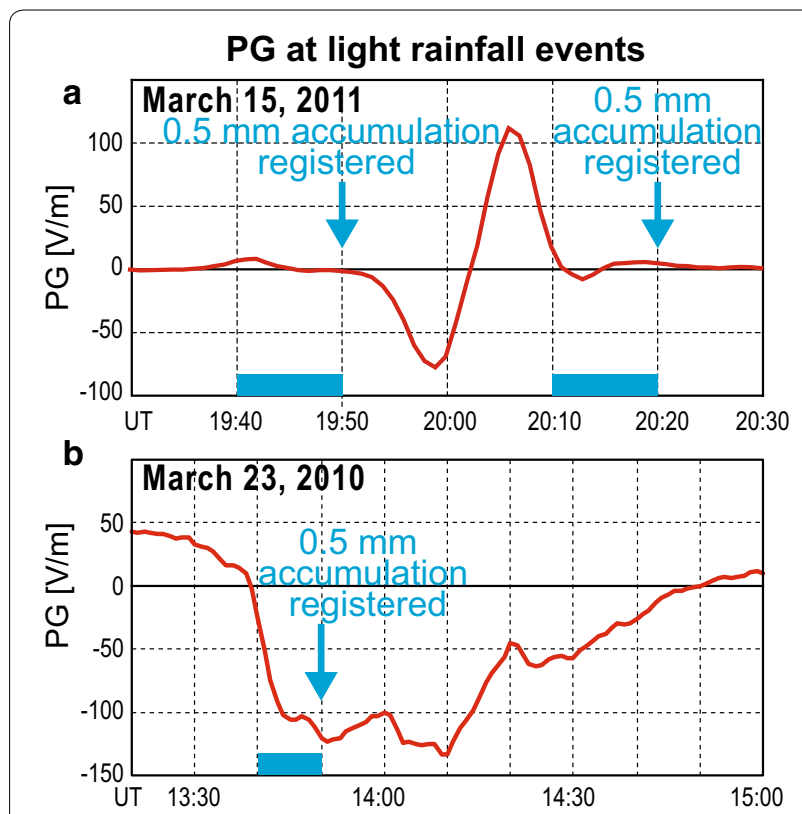

Fig. 2 One-minute averaged values of $P G$ around isolated light rain (0.5 mm in 10-min accumulation) on a March 15, 2011, and b March 23, 2010. Horizontal blue lines and vertical blue arrows denote the rain periods and the registration timing of the accumulation rain
$2000 \mathrm{~V} / \mathrm{m}$ changes in about $30 \mathrm{~s}$, guaranteeing that the PG measurements react to changes in the cloud charges with $<1$ min resolution.

At Kakioka, the rain data was also measured using the unmanned accumulation method every $10 \mathrm{~min}$ at 0.5 $\mathrm{mm}$ resolution to register rainfall accumulation without measuring other weather information. Therefore, light rain of less than $0.5 \mathrm{~mm}$ was not registered until the accumulated value reached $0.5 \mathrm{~mm}$ (cf. Fig. 2). For example, continuous rain of $0.3 \mathrm{~mm} / 10 \mathrm{~min}$ will be registered in every other slices of $10 \mathrm{~min}$.

Figure 3 shows distribution of PG values for different rain conditions at 10 -min resolution at Kakioka from March 13 to April 30 for 2006-2015, divided into 2011 and the other years. We classified the weather conditions by the 10-min values of the registered rainfall; the figure shows three categories (see figure captions). We did not show strong rain cases (registering $\geq 1.0 \mathrm{~mm}$ in $10 \mathrm{~min}$ ) because they are rare with insufficient statistics for 2011. For the $<0.5 \mathrm{~mm}$ category, the difference between 2011 and the average of the other years is not significant compared to the annual difference, e.g., 2009 (not shown here) had a low level similar to 2011, although it is larger than the statistical error calculated from the standard deviation. Under these restrictions, Fig. 3 shows that, after the FNPP1 accident, 10-min average values of the rain-time PG decreased compared to the other years with peak values unchanged, supporting the difference shown in Fig. 2 (10-min averaged negative PG values for the same peak values are reduced in 2011).

Here, we examined simple distribution of the 10-min PG values to improve the statistical reliability. Ideally, data would be subdivided according to meteorological properties (e.g., wind, cloud conditions, lightning conditions), but that makes statistics unreliable in the present event study. Fortunately, balloon and ground data at Tsukuba (20 km from Kakioka) shows that the rain-time wind velocities at ground and cloud altitudes from March 13 to April 30 in 2011 were about the average level of the same period of 2006-2015. Here, we treated all 10-min data from all weather conditions together when the 10 -min bins included PG peaks of $<-100$ or $>+150 \mathrm{~V} / \mathrm{m}$ in the 1 -min values, after removing minor stagnating peaks (less than $1 \%$ of the peak values from the trend) on the way or from the other larger peaks.

Figure 4 shows the peak value distributions (upper panel) and 10-min averages around these peak values (lower panel), using 1-min resolution PG values. The peaks are counted in the logarithmic bins (20\% stepping) of the PG peak values. The occurrence rate of the PG peak values immediately after the FNPP1 accident is generally similar to those for the other years in Fig. 4a. However, the average PG over 10 min compared to minimum 
PG distribution vs Rain at Kakioka, Mar 13 - April 30, 2006-2015

a 10-min average

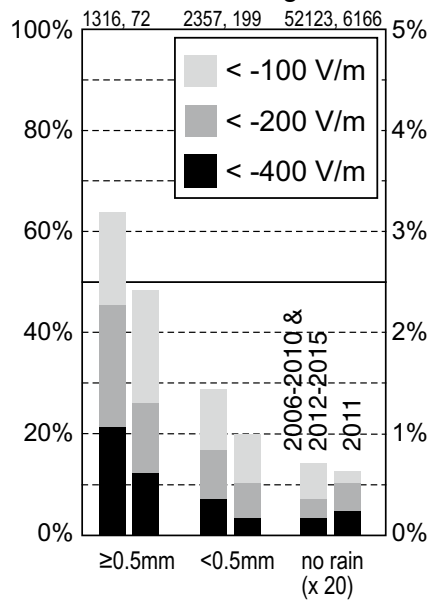

b minimum (1-min value)

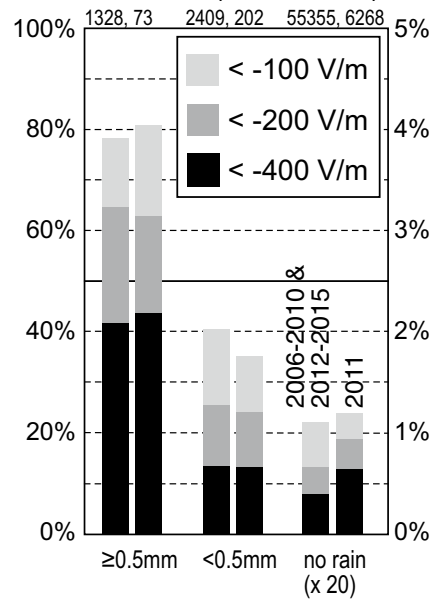

C maximum (1-min value)

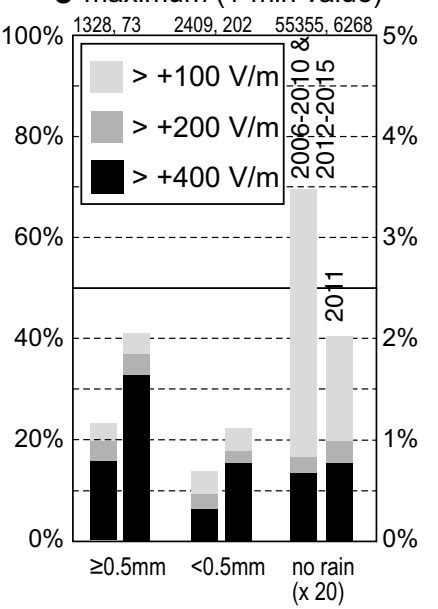

Fig. 3 Observation probability of negative potential gradient (PG) near or during the rain time at Kakioka, $150 \mathrm{~km}$ southwest of the Fukushima Dai-ichi Nuclear Power Plant (FNPP1). The 1-min PG date in 10 min bins is used to classify according to a 10-min average, $\mathbf{b}$ minute values at negative peak, and $\mathbf{c}$ minute values at positive peak, into $<-400,-400$ to $-200,-200$ to $-100,+100$ to $+200,+200$ to +400 , and $>+400 \mathrm{~V} / \mathrm{m}$ for different rain conditions: continuous rain $(\geq 0.5 \mathrm{~mm})$, either light rain $(<0.5 \mathrm{~mm})$ or within $10 \mathrm{~min}$ before the rain, and no rain over $30 \mathrm{~min}$ before or after. Since the percentage is very low for no rain cases, the unit is changed in the graph (right axis). The rain record is obtained by an accumulation method every 10 min (see text for detail). The data are compared between immediately after the FNPP1 accident (13 March-30 April, 2011) and the same period (13 March-30 April) at different years (2006-2015). Total numbers of 10-min bins are given at the top of each bar
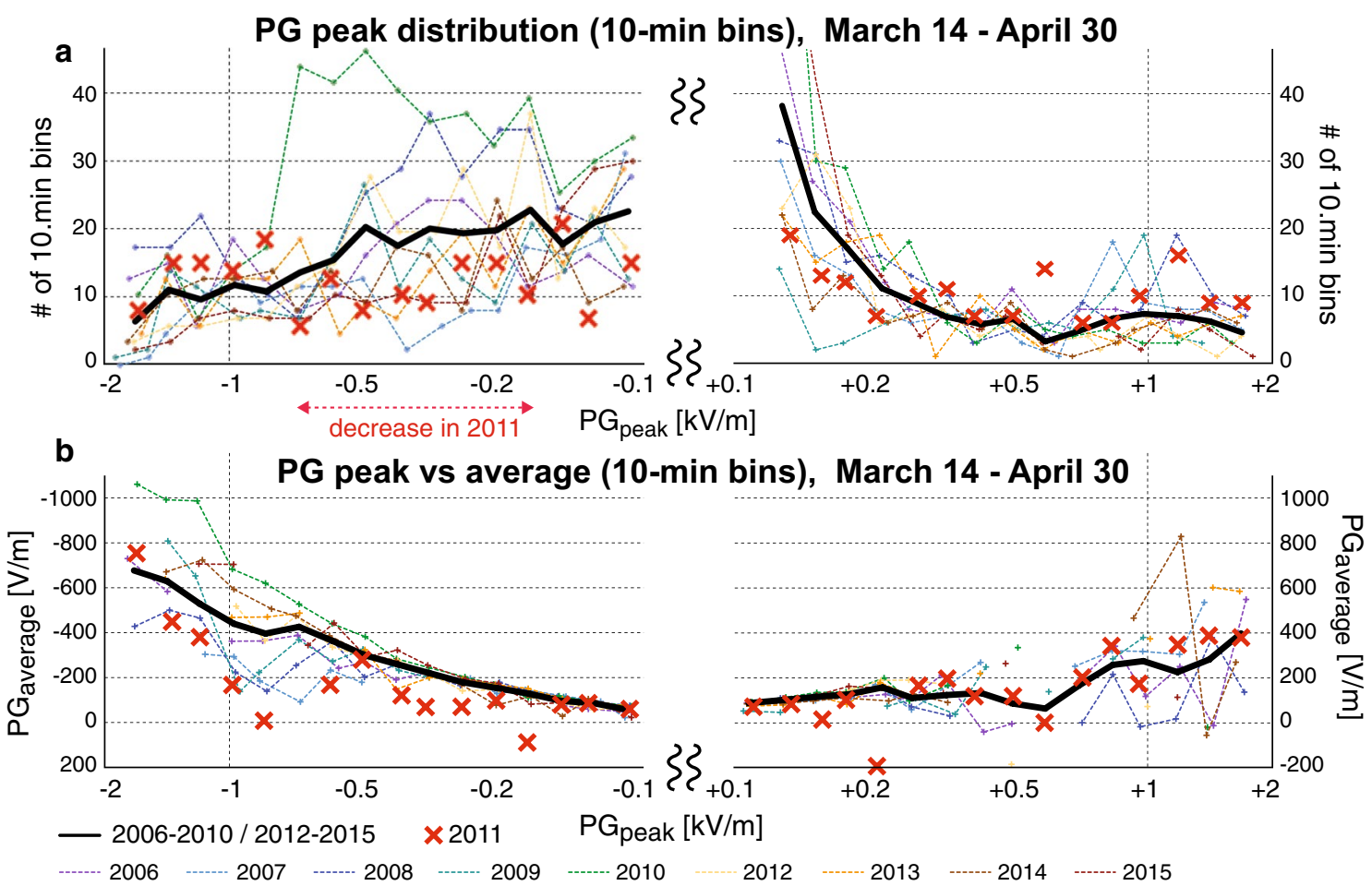

Fig. 4 a Distribution of PG peak minute values during 50 days in the same season for 2006-2015. PG peaks are registered every 10 min, and numbers of these peaks within bins that are defined in logarithmic scale (20\% stepping) are plotted, such that exponential distribution becomes a constant line, $\mathbf{b} 10$-min averages for each bin of PG peaks. A larger difference means quick rise and/or quick decay from the peak values. In both panels, red crosses are for 2011, dashed lines are for the other years, and thick line is for the average 
1-min values was somewhat lower in 2011 than all the other years for PG peak values of $<-200 \mathrm{~V} / \mathrm{m}$, suggesting that the rain-time difference shown in Fig. 3 could be real. This result also suggests that the ground PG values changed more quickly around the peak values in 2011 than the other years.

To examine how quickly the PG value reaches its negative peak or decays from it, Fig. $5 \mathrm{a}-\mathrm{l}$ shows the time profile around the negative peaks with $\mathrm{PG}_{\text {peak }}<-100 \mathrm{~V} / \mathrm{m}$. Relative PG value against the peak value at each minute within \pm 5 min from the peak was averaged for three periods each year: (a)-(d) February 1-March 10, (e)(h) March 14-April 30, and (i)-(l) May 1-31. For 2011, these periods correspond to: before the FNPP1 accident, periods of high radioactivity in the atmosphere, and sufficiently decreased radioactivity, respectively. To minimize the effect of variance with short timescale, we used the time range when the PG increase or decreased around the peak was monotonic. Therefore, different data points (minutes) have different numbers of samples, and those with fewer than seven samples are not plotted.

When the radioactive particles were floating, immediately after the FNPP1 accident (March 14-April 30, 2011), the PG increased toward and decreased from the negative peak faster than in the other years, as shown in Fig. $5 \mathrm{e}-\mathrm{h}$. The time constant (1/e time) changed from about 4-5 min to less than $3 \mathrm{~min}$ for both development and decay. The result is the same if we examine the characteristic time at which more than $50 \%$ of events have PG values less than the $1 / e$ of the peak value. The difference between 2011 and the other years is most significant for the PG peak values of -400 to $-200 \mathrm{~V} / \mathrm{m}$ (with much lower ratio in 2011 than all the other years), but such a difference is also detectable for other PG peak values compared with the average of 2006-2015.

The shorter timescale for 2011 is not seen before the FNPP1 accident (Fig. 5a-d), or in May, during which the 2011 profile is within the range of the annual variation (Fig. 5i-l). In May, the floating radioactive particles (which may have included both new inflow from the FNPP1 area and re-suspensions from other areas) decreased significantly while the surface contamination level stayed at the same level (Yamauchi et al. 2012; Yamauchi 2012; Kanai 2012; Kita et al. 2013). The profiles for the normal years are different between May and March-April because of the different weather systems, but the March-April 2011 profile is still at the lowest limit of the annual variation for May, and outside the annual variation in Fig. 5g, h. Also, the average time profiles lie around the upper limit of the standard deviation of 2011 for each minute, and hence the anomaly in 2011 is most likely real.

For reference, Fig. 5m-o shows the change from the positive peaks, in which we do not see clear differences between 2011 and the other years when $\mathrm{PG}_{\text {peak }}>+400 \mathrm{~V} / \mathrm{m}$. On the other hand, for the lower PG peak values $(<+200 \mathrm{~V} / \mathrm{m})$, the short time constant in 2011 is most likely an artifact from the difference in the fair-weather PG. (Start value and decay destination are $\sim 0 \mathrm{~V} / \mathrm{m}$ for 2011 and $\sim+60 \mathrm{~V} / \mathrm{m}$ for the other years.) The change in the offset value in turn reinforces the shortening of the timescale for negative PG in 2011.

\section{Discussion}

Figures $4 \mathrm{~b}$ and 5 indicate that timescale toward/from the PG negative peak is shortened when the floating radioactive particles from the FNPP1 increased the atmospheric conductivity $\sigma$ through the enhanced ionization rate. While the difference in PG behavior might simply have come from coincidental difference in the cloud and atmospheric condition as mentioned above, we consider a scenario by increased ionizing radiation.

In the introduction, we proposed three mechanisms in which the increased atmospheric radioactivity may influence the PG behavior (enhanced discharge rate, Debye shielding, and dielectric shielding). In all scenarios, we must also consider both (a) the local development case (ground-charge distance is constant) and (b) the moving case (ground-charge distance changes by either horizontal convection like Fig. 1 or vertical oscillation).

(1) The shortened $\tau_{d}$ scenario predicts asymmetric change between rise (becomes slower) and decay (becomes faster) in both cases. (2) The Debye shielding scenario depends on the local ion density near the cloud charge within the Debye shielding distance, causing the reduction rate of PG rather constant at all phases. In other words, it predicts smaller PG (absolute) values with the same timescale in both cases. (3) The dielectric shielding depends largely on the total numbers of shielding ions and hence, the distance between the cloud charges and the ground. Therefore, we expect different time profiles, i.e., (a) constant suppression rate of PG without change in the timescale if the cloud charges develop/decay locally and (b) quick rise and quick decay (symmetric shortening of the timescale) if the cloud charges are moving, like Fig. 1.

In all scenarios, 10-20-min averaged PG values centered at the PG peak are expected to be smaller during the 50 days after the FNPP1 accident as compared to the same period of the other years, as shown in Fig. 3. On the other hand, the second scenario is not consistent with Figs. 4 and 5 , and we can dismiss it. The first scenario predicts shorter decay time from the peak but not the shortening of rising time toward the peak, as is shown in Fig. $5 \mathrm{~g}$. Therefore, the observation cannot be explained by this scenario alone. The third scenario (dielectric shielding) predicts quick rise and quick decay for the moving-charge case. 


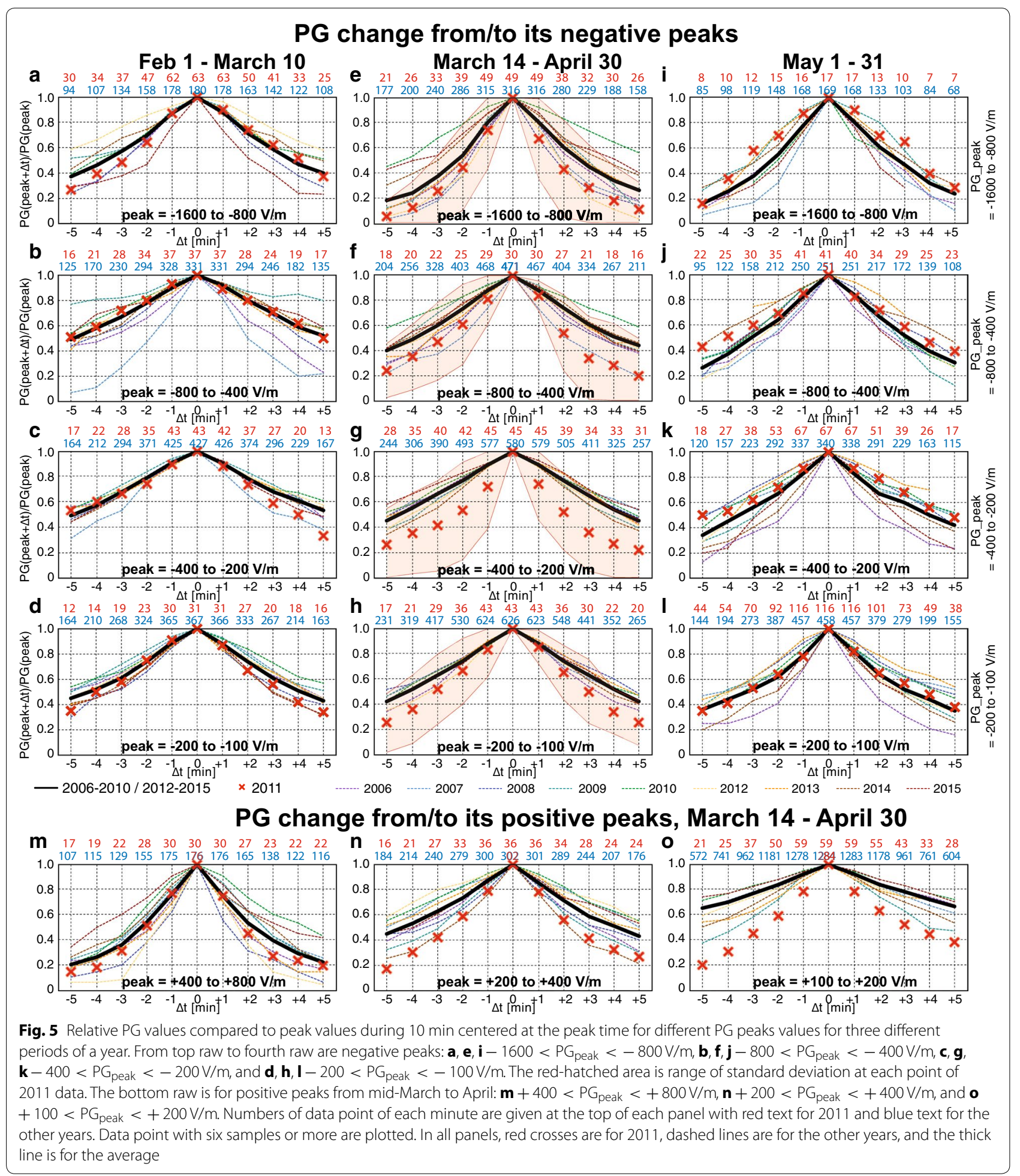

Thus, we have at least one scenario that explains the observation (particularly Fig. 5g), i.e., electrostatic (dielectric) shielding of cloud charge moving such that distance (and total numbers of ions) between the charge and the ground first decreased to the minimum distance and then increased, as illustrated in Fig. 1. Although Fig. 1 illustrates horizontal motion, vertical oscillation is also possible because the average decay time of $4-5 \mathrm{~min}$ in the 
normal years corresponds to Brunt-Vaisala frequency of vertical oscillation of air parcel in a stable atmosphere.

Nevertheless, we still cannot dismiss the first scenario because the observed shortening of the decay time by a factor of nearly 2 indicates an enhancement of the ion density $n_{i}$ by a factor of nearly 2 , a realistic number, in this scenario. We have not found a scenario that also predicts faster rising time toward the PG peak, i.e., a mechanism that enhance the accumulation rate of the cloud charges (2-5 min in normal condition) when the surrounding radioactivity is enhanced. If such a mechanism is found, this scenario also becomes a plausible explanation.

Figures $4 \mathrm{~b}$ and 5 also suggest that the timescale toward/ from the PG positive peak is unchanged in 2011. This can be explained by the tendency that positive charges are located at higher altitude in electrified clouds (e.g., Israel 1973). If the radioactive particles from the FNPP1 did not spread above the altitude of negative charges, the electrostatic shielding by ions at higher altitudes is not very effective. In other words, the different PG profiles between around the positive peaks and around the negative peaks may hint the altitude extension of the floating radioactive particles.

Since rainfall sweeps the floating dusts from the air, we expect that the effect is strongest during light rain or just before the rain. Unfortunately, statistics for heavy rain are insufficient (cf. Fig. 3). Also the radar data of clouds (Yatagai et al. 2012) is not clear over Kakioka due to very low spatial resolution and sensitivity (Yatagai, private communication, 2016). Therefore, we can only point out that the difference between 2011 and the other years in the PG distribution during light rain $(\leq 0.5 \mathrm{~mm})$ in Fig. 3 is consistent with this scenario. The washing of the floating radionuclides might also explain the largest difference in the time constant (Fig. 5) between 2011 and other years at moderately large negative PG $(-200$ to $-400 \mathrm{~V} / \mathrm{m})$, because the largest negative PG (e.g., $<-800 \mathrm{~V} / \mathrm{m}$ ) are mainly observed during heavy rain.

Finally, we consider the effect of finite re-distribution time of PG at different altitudes through conduction current (the $\vec{J}$ terms instead of the $\partial \vec{D} / \partial t$ term in the Ampere's law) in response to the cloud charge distribution, because this takes longer time near ground due to the much lower conductivity (or dielectric constant) than in the cloud. If the conductivity near the ground increases, this effect makes the response to semi-stable cloud charge (that stays for a half to a few minutes) faster, resulting measuring higher PG peak absolute values at ground for the same cloud charge. This scenario qualitatively explains the rising part of Fig. $5 \mathrm{~g}$, and is also consistent with Fig. 4a in which the distribution of peak PG values can be interpreted as being shifted toward higher absolute values. However, this scenario does not explain
Fig. $5 \mathrm{~m}$ although negative charges are located closer to the ground (expecting faster response) than the positive charges. Furthermore, if this mechanism is significant, we expect quick rise and slow decay in the normal year, but such asymmetry is not shown in Fig. 5. Therefore, this mechanism is unlikely a major factor to shorten the timescale (Fig. 5g), although it should contribute some.

\section{Conclusions}

One-minute resolution PG data and 10-min resolution weather data at Kakioka, $150 \mathrm{~km}$ southwest of the Fukushima Dai-ichi Nuclear Power Plant were analyzed during the February-May periods of 2006-2015 to find the effect of floating radioactive particles on PG under rain clouds. The data revealed that the characteristic time toward and from negative PG peaks of $<-200 \mathrm{~V} / \mathrm{m}$ shortened ( $\leq 3 \mathrm{~min}$ for both development and decay) during March-April 2011 as compared to the same period of the other years (4-5 $\mathrm{min}$ ) beyond the annual difference, while no significant difference is seen in February and May. Assuming the atmospheric conditions when electrified clouds present were the same, this result can be attributed to enhanced electrostatic (dielectric) shielding by the increased ion density even in extremely weakly ionized plasma with high collision frequency, although we cannot dismiss the effect of discharge rate enhancement (shortening of $\tau_{d}$ ) by the increased electric conductivity for the decay part. The result opens up new possibility of monitoring method for radioactivity in the atmosphere in case of nuclear accidents in the future.

\section{Authors' contributions}

MY conducted the analyses and prepared the manuscript, MT contributed to the presentation and interpretations of the result, and SN and his team conducted the PG measurement and prepared the 1-s and 1-min resolution data. All authors read and approved the final manuscript.

\section{Author details}

${ }^{1}$ Swedish Institute of Space Physics, Box 812, 98128 Kiruna, Sweden. ${ }^{2}$ Data Analysis Center for Geomagnetism and Space Magnetism, Graduate School of Science, Kyoto University, Kyoto, Japan. ${ }^{3}$ Kakioka Magnetic Observatory, Japan Meteorological Agency, Ishioka, Japan.

\section{Acknowledgements}

We thank M. Makino for the useful comments on the effect of the atmospheric conductivity, and T. Toya and T. Owada for their advice on the atmospheric electricity (PG) data obtained from the Kakioka Geomagnetic Observatory. The lead author (MY) wishes to thank Swedish programs for physically disabled individuals, which have made it possible for him to conduct this work.

\section{Competing interests}

The authors declare that they have no competing interests.

\section{Availability of data and materials}

The PG and weather data are were provided by the Japan Meteorological Agency and publicly available at http://www.kakioka-jma.go.jp/obsdata/metadata/en/ and http://www.data.jma.go.jp/gmd/risk/obsdl/index.php. Analyses program is found at http://www2.irf.se/\%7Eyamau/code_public/PG_rain/.

Ethics approval and consent to participate

Not applicable. 
Funding

Not applicable.

\section{Publisher's Note}

Springer Nature remains neutral with regard to jurisdictional claims in published maps and institutional affiliations.

Received: 31 July 2017 Accepted: 4 January 2018

Published online: 21 March 2018

\section{References}

Debye P, Huckel E (1923) The theory of electrolytes. I. Lowering of freezing point and related phenomena. Phys Z 24:185-206

Fukushima University (2011) Press release, 11 May. http://www.sss.fukushimau.ac.jp/sonde_data

Harris DL (1955) Effects of radioactive debris from nuclear explosions on the electrical conductivity of the lower atmosphere. J Geophys Res 60:45-52

Harrison RG, Carslaw KS (2003) Ion-aerosol-cloud processes in the lower atmosphere. Rev Geophys 41 (3):1012. https://doi. org/10.1029/2002RG000114

Israel H (1973) Atmospheric electricity. Fields, charges and currents, vol 2. Published for the National Science Foundation by the Israel Program for Scientific Translations, ISBN 0706511298

Israelsson S, Knudsen E (1986) Effect of radioactive fallout from a nuclear power plant accident on electrical parameters. J Geophys Res 91:11909-11910

Israelsson S, Schutte T, Pisler E, Lundquist S (1987) Increase occurrence of lightning flashes in Sweden during 1986. J Geophys Res 92:10996-10998

Kanai Y (2012) Monitoring of aerosols in Tsukuba after Fukushima Nuclear Power Plant incident in 2011. J Environ Radioact 111:33-37

Kita K, Kasahara R, Tanaka M, Sato K, Demizu H et al (2013) Variation of atmospheric concentrations of I-131, Cs-134 and Cs-137 observed over eastern
Japan: contribution of leakage from Fukushima Dai-ichi Nuclear Power Plant and secondary emission from soil and vegetation. Geophys Res Abstr 15:EGU2013-5090

Markson R (2007) The global circuit intensity: its measurement and variation over the last 50 years. Bull Am Meteorol Soc 88:223-241

NISA (2011) The Accident at TEPCO's Fukushima nuclear power stations, Report of Japanese government to the IAEA ministerial conference on nuclear safety, 7 June 2011, chapter VI. Press release by Nuclear and Industrial Safety Agency (NISA), Japan. http://www.iaea.org/newscenter/ focus/fukushima/japan-report/

Rycroft MJ, Harrison RG, Nicoll KA, Mareev EA (2008) An overview of Earth's global electric circuit and atmospheric conductivity. Space Sci Rev 137:83-105. https://doi.org/10.1007/s11214-008-9368-6

Shigeno N, Takizawa T, Itoh N, Yokoyama M, Owada T (2001) Preliminary test for atmospheric electricity measured using an electrostatic sensor. Gijutsu Hookoku 112:8-13

Takeda M, Yamauchi M, Makino M, Owada T (2011) Initial effect of the Fukushima accident on atmospheric electricity. Geophys Res Lett 38:L15811. https://doi.org/10.1029/2011GL048511

Tuomi TJ (1988) Observations of atmospheric electricity 1986. Geophysical Publications 7:551.506.1. Finish Meteorological Institute, Helsinki

Williams ER (2009) The global electrical circuit: a review. Atmos Res 91:140152. https://doi.org/10.1016/j.atmosres.2008.05.018

Yamauchi M (2012) Secondary wind transport of radioactive materials after the Fukushima accident. Earth Planets Space 64(1):e1-e4. https://doi. org/10.5047/eps.2012.01.002

Yamauchi M, Takeda M, Makino M, Owada T, Miyagi I (2012) Settlement process of radioactive dust to the ground inferred by the atmospheric electric field measurement. Ann Geophys 30:49-56. https://doi.org/10.5194/ angeo-30-49-2012

Yatagai A, Kamiguchi K, Arakawa O, Hamada A, Yasutomi N, Kitoh A (2012) APHRODITE: constructing a long-term daily gridded precipitation dataset for Asia based on a dense network of rain gauges. Bull Am Meteorol Soc 93(9):1401-1415. https://doi.org/10.1175/BAMS-D-11-00122.1

\section{Submit your manuscript to a SpringerOpen ${ }^{\circ}$ journal and benefit from:}

- Convenient online submission

- Rigorous peer review

- Open access: articles freely available online

- High visibility within the field

- Retaining the copyright to your article

Submit your next manuscript at $>$ springeropen.com 